\title{
A Low Serum CCL4/MIP-1 $\beta$ Level May Predict a Severe Asthmatic Responsiveness to Mepolizumab
}

\author{
Maho Suzukawa ${ }^{1,2}$, Nobuharu Ohshima ${ }^{3}$, Hiroyuki Tashimo ${ }^{1}$, Isao Asari ${ }^{2}$, \\ Nobuyuki Kobayashi ${ }^{1}$, Shunsuke Shoji ${ }^{1}$, Shigeto Tohma ${ }^{1}$ and Ken Ohta ${ }^{1,2,4}$
}

\begin{abstract}
:
Objective Mepolizumab, a humanized anti-interleukin-5 monoclonal antibody, is effective for treating eosinophilic severe asthma. However, there is a need for more biomarkers that can predict the patient response to mepolizumab before starting therapy. This study aimed to identify a new biomarker in the serum that is able to accurately predict the responsiveness to mepolizumab.

Methods This study enrolled 11 patients who had all been diagnosed with severe eosinophilic asthma and were then administered mepolizumab every 4 weeks for at least 4 months. Blood samples were collected, and pulmonary function tests and questionnaires were administered at baseline and after 4, 8 and 16 weeks of treatment. The response to mepolizumab was then assessed based on the difference in the Asthma Quality of Life Questionnaire (AQLQ) score after 16 weeks of mepolizumab therapy compared with that at baseline. Patients with an increase in the AQLQ score of more than 0.5 were defined as responders. The cytokine levels in the blood were measured by LUMINEX 200 and ELISA.

Results There were 6 responders and 5 non-responders. The responders showed a significantly lower serum level of chemokine (C-C motif) ligand 4/macrophage inflammatory protein-1 $\beta$ (CCL4/MIP-1 $\beta$ ) at baseline compared to the non-responders. Receiver operating characteristic curves to distinguish responders from nonresponders using the baseline serum CCL4/MIP-1 $\beta$ level showed a good area under the curve of 0.9 . The non-responders showed a significant increase in the level of CCL4/MIP-1 $\beta$ after 4 weeks compared to the baseline.

Conclusion A low baseline serum CCL4/MIP-1 $\beta$ level may be useful for predicting a good mepolizumab response in severe eosinophilic asthma.
\end{abstract}

Key words: mepolizumab, asthma, eosinophils, CCL4/MIP-1 $\beta$

(Intern Med 59: 2849-2855, 2020)

(DOI: 10.2169/internalmedicine.5159-20)

\section{Introduction}

Severe asthma patients are reported to make up from 5 to $10 \%$ of asthma patients, and they have a very poor QOL $(1,2)$. Severe asthma has negative socioeconomic effects because it requires special medical care, including hospitalization and intensive care of such patients; this disease therefore remains a big problem for respiratory medicine clinics (3). However, recent advances in the treatment of asthma, in addition to the earlier development of inhaler therapy, have succeeded in improving the QOL of even severe asthma patients. Today, we have a few biological drugs that neutralize the inflammatory molecules involved in the pathogenesis of type 2 inflammation, namely, IgE, interleukin-5 (IL-5), IL-4 and IL-13, and there are several drugs to choose from for treating severe asthma besides systemic steroid use (4).

On the other hand, physicians often agonize over which biologic to choose as a first-line therapy for each pa-

\footnotetext{
${ }^{1}$ Asthma, Allergy and Rheumatology Center, National Hospital Organization Tokyo National Hospital, Japan, ${ }^{2}$ Clinical Research Center, National Hospital Organization Tokyo National Hospital, Japan, ${ }^{3}$ Center for Pulmonary Diseases, National Hospital Organization Tokyo National Hospital, Japan and ${ }^{4}$ Department of Respiratory Medicine, Fukujuji Hospital, Japan Anti-Tuberculosis Association, Japan Received: April 23, 2020; Accepted: June 7, 2020; Advance Publication by J-STAGE: July 21, 2020 Correspondence to Dr. Maho Suzukawa, fueta-tky@umin.ac.jp
} 
tient $(5,6)$. Indeed, there are few biomarkers to suggest which drug would most benefit the patient to be treated. For example, an analysis of the EXTRA omalizumab study showed that the peripheral blood eosinophil count and fractional exhaled nitric oxide (FeNO) level at baseline may predict responsiveness to omalizumab as assessed by the rate of asthma exacerbation during the first year of treatment (7). In addition, we recently reported that the baseline serum C-X-C motif chemokine 10 (CXCL10) and IL-12 levels may be good biomarkers for predicting the responsiveness of severe asthmatics to omalizumab (8). In addition to the difficulty in deciding which biological drug to use, biologics often show a gradual effect so that patients have to continue receiving them for a long time to clearly identify their efficacy. The guidelines recommend trying them for at least four months to see if the asthma symptoms improve $(9,10)$. Since these drugs are expensive and sometimes do not provide commensurate advantages to the patients, there is a strong need for good biomarkers that predict the patients responsiveness to them.

One biologic used for severe asthma is mepolizumab, a humanized anti-IL-5 monoclonal antibody that is characterized by its efficacy in treating eosinophilic severe asthma (11-13). The baseline blood eosinophil count is reported to predict responsiveness to mepolizumab therapy $(14,15)$. In addition, we showed that the baseline Siglec-8 expression may predict responsiveness to mepolizumab (16). Since IL-5 plays a pivotal role in the pathogenesis of eosinophilic asthma as one of the most potent activators of eosinophils, it is logical that the baseline blood eosinophil count would predict responsiveness to mepolizumab. Similarly, since Siglec-8 has the opposite effect on eosinophil survival to that of IL-5, it is easy to imagine that the serum level of soluble Siglec- 8 can also predict responsiveness to mepolizumab.

A greater number of reliable biomarkers for predicting responsiveness to mepolizumab would help physicians decide which drug should be used for each patient. Therefore, we conducted the present study with the aim of identifying new biomarkers in the blood at baseline, and we found that a low baseline serum chemokine (C-C motif) ligand 4/macrophage inflammatory protein-1 $\beta$ (CCL4/MIP-1 $\beta$ ) level was useful for predicting a good mepolizumab response in severe eosinophilic asthmatics.

\section{Materials and Methods}

\section{Subjects}

The subjects consisted of 11 patients who had been diagnosed with severe eosinophilic asthma and were then treated with mepolizumab for at least 4 months. The patients were enrolled between August 2016 and July 2017 at National Hospital Organization Tokyo National Hospital. Asthma was diagnosed according to the criteria of the American Thoracic Society (ATS). The study protocol was approved by the Eth- ics Committee of Tokyo National Hospital, and written informed consent was obtained from each of the subjects.

\section{Data and sample collection}

All the patients were administered mepolizumab by sc injection every 4 weeks for at least 4 months. Blood samples were collected at baseline and after 4,8 and 16 weeks of treatment. Serum was separated by centrifugation and kept frozen at $-20^{\circ} \mathrm{C}$ until measurement of the serum cytokine levels. The following clinical variables were determined at baseline, and after 4, 8 and 16 weeks of mepolizumab therapy: body mass index (BMI), peripheral blood eosinophil count, serum IgE level, pre-bronchodilator pulmonary function, FeNO level, Asthma Control Questionnaire (ACQ) score, Asthma Quality of Life Questionnaire (AQLQ) score and visual analog scale (VAS) of asthma symptoms.

\section{Responders and non-responders}

The response to mepolizumab was assessed based on the change in the AQLQ score after 16 weeks of mepolizumab treatment relative to the baseline. Patients with an increase of more than 0.5 were classified as responders, whereas others were classified as non-responders.

\section{Serum cytokine levels}

Serum samples were assayed for 10 cytokines [IL-4, -5, -9, -12; CXCL10/interferon gamma-induced protein 10 (IP10), CCL11/eotaxin, interferon (IFN)- $\gamma$, CCL4/MIP-1 $\beta$, platelet-derived growth factor (PDGF)-BB and vascular endothelial growth factor (VEGF)] by using Human Bio-Plex Pro (BioRad Laboratories, Hercules, USA) and LUMINEX 200 (Luminex, Austin, USA) according to the manufacturers' instructions. IL-33 (assay range: 6.3-400 pg/mL), thymic stromal lymphopoietin (TSLP) (assay range: 31.2-2,000 $\mathrm{pg} / \mathrm{mL}$ ) and chitinase 3-like 1 (CHI3 L1) (assay range: 62.5$4,000 \mathrm{pg} / \mathrm{mL}$ ) were measured using ELISA kits from R\&D Systems, and periostin (assay range: 20-2,000 pg/mL) was measured using an ELISA kit from Shino-Test (Tokyo, Japan), each according to the manufacturer's instructions.

\section{Statistical analyses}

Univariate analyses were performed using Student's $t$ test and the Mann-Whitney $U$ test. Subgroups of patients were compared using Fisher's exact test. Changes in the overall AQLQ score and the levels of biomarkers at different time points were analyzed by the 2-way analysis of variance (ANOVA). A receiver operating characteristic (ROC) analysis was performed, and the area under each ROC curve (AUC) was calculated. A two-tailed P-value of less than 5\% was considered to be statistically significant. All statistical analyses were performed using GraphPad Prism version 7.0 for MAC OS X (GraphPad Software, San Diego, USA). 
(A)

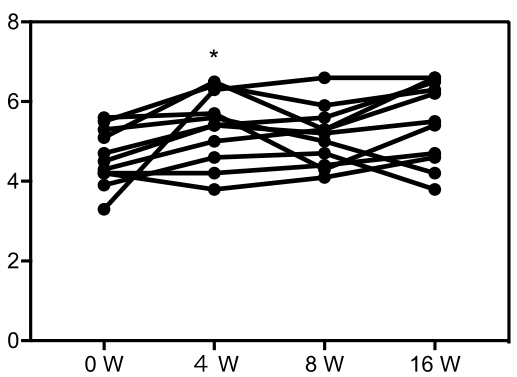

(B)

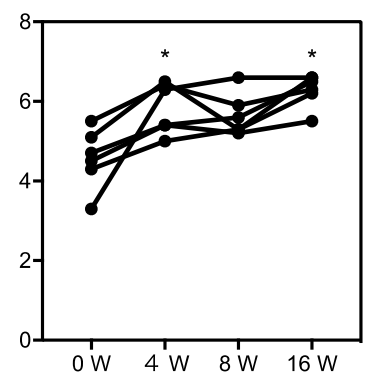

(C)

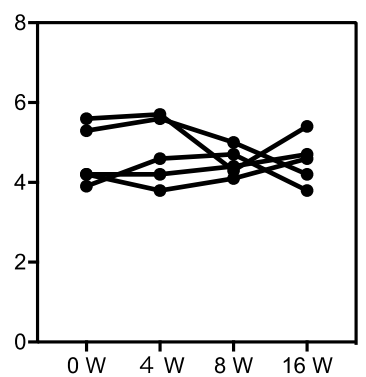

Figure 1. Overall AQLQ scores during mepolizumab therapy. The overall AQLQ scores during mepolizumab therapy are shown for all patients (A), responders (B) and non-responders (C). Responders and non-responders were defined by the increase in the AQLQ score at 16 weeks from baseline (i.e., responders, $0.5<$; and non-responders, $\leq \mathbf{0 . 5}$ ). * ${ }^{*}<0.05$ vs. AQLQ overall score at baseline. AQLQ: Asthma Quality of Life Questionnaire

Table 1. Baseline Characteristics of the Patients, Stratified by the Change in the AQLQ Score at 4 Months.

\begin{tabular}{lccc}
\hline \multicolumn{1}{c}{ Characteristic } & Responders & Non-responders & p value \\
\hline $\mathrm{n}$ & 6 & 5 & \\
Age (years) & $59.67 \pm 17.42$ & $53.4 \pm 23.31$ & 0.62 \\
Gender (male/female) & $3 / 3$ & $0 / 5$ & \\
BMI $\left(\mathrm{kg} / \mathrm{m}^{2}\right)$ & $20.98 \pm 1.67$ & $24.26 \pm 6.63$ & 0.27 \\
Disease duration (years) & $26.5 \pm 17.83$ & $16 \pm 9.59$ & 0.27 \\
Smoking index & $165 \pm 159.1$ & $80 \pm 80$ & 0.67 \\
FeNO level (ppb) & $112.8 \pm 69.14$ & $57.18 \pm 39.45$ & 0.20 \\
Blood eosinophil count $(/ \mu \mathrm{L})$ & $590.3 \pm 346.5$ & $467 \pm 303.7$ & 0.55 \\
Blood neutrophil count $(/ \mu \mathrm{L})$ & $4,082 \pm 510.5$ & $4,543 \pm 1,063$ & 0.69 \\
Total serum IgE (IU/mL) & $137.3 \pm 99.5$ & $162.2 \pm 207.3$ & 0.80 \\
FEV $(\%$ predicted) & $65.3 \pm 20.7$ & $75.6 \pm 15.1$ & 0.38 \\
FEV $(\mathrm{L})$ & $1.46 \pm 0.61$ & $1.49 \pm 0.54$ & 0.94 \\
AQLQ overall score & $4.6 \pm 0.76$ & $4.6 \pm 0.76$ & 0.88 \\
Asthma VAS score $(\mathrm{cm})$ & $3.3 \pm 3.61$ & $5.46 \pm 3.32$ & 0.33 \\
OCS use $(\%)$ & $1(16.7)$ & $2(40)$ & \\
High-dose ICS use $(\%)$ & $6(100)$ & $5(100)$ & \\
LABA use $(\%)$ & $6(100)$ & $5(100)$ & \\
LAMA use $(\%)$ & $3(50)$ & $2(40)$ & \\
LTRA use $(\%)$ & $5(83.3)$ & $4(80)$ & \\
Theophylline use $(\%)$ & $4(66.7)$ & $4(80)$ & \\
Use of other biologics within previous three months $(\%)$ & 1, omalizumab $(16.7)$ & 1, omalizumab $(20)$ & \\
\hline
\end{tabular}

Values are presented as the mean \pm SD.

AQLQ: Asthma Quality of Life Questionnaire, FEV 1 : forced expiratory volume in $1 \mathrm{~s}$, OCS: oral corticosteroid, ICS: inhaled corticosteroid, LABA: long-acting beta-agonists, LAMA: long-acting muscarinic antagonists, LTRA: leukotriene receptor antagonists

\section{Results}

\section{Baseline characteristics of the patients, stratified by their response to mepolizumab therapy}

All 11 patients with eosinophilic severe asthma showed a significant improvement in the AQLQ score after 4 weeks of mepolizumab therapy (Fig. 1A). However, after both 8 and 16 weeks, although some of the participants showed an im- provement in terms of an elevated AQLQ score, none of them showed a significant improvement in the overall AQLQ score relative to the baseline. Therefore, we divided the patients into responders and non-responders based on an improvement in the AQLQ score of more or less than 0.5 after 16 weeks (Fig. 1B, C). There were 6 responders and 5 non-responders, and the responders showed a significant increase in the AQLQ score after both 4 and 16 weeks (Fig. 1B) while the non-responders showed no apparent change in the AQLQ score (Fig. 1C). 
Table 2. Clinical Outcomes of the Patients, Stratified by the Change in the AQLQ Score after 4 Months.

\begin{tabular}{|c|c|c|c|}
\hline Characteristic & Responders & Non-responders & $\mathrm{p}$ value \\
\hline $\mathrm{n}$ & 6 & 5 & \\
\hline$\delta$ ACQ score (Baseline-at 4 months) & $1.2 \pm 1.11$ & $-0.2 \pm 0.60$ & 0.03 \\
\hline$\delta$ FeNO level (ppb, at 4 months-baseline) & $-48.7 \pm 47.02$ & $-6.33 \pm 20.46$ & 0.14 \\
\hline$\delta$ blood eosinophil count ( $/ \mu \mathrm{L}$, at 4 months-baseline) & $-546.5 \pm 348.3$ & $-390.4 \pm 261.3$ & 0.43 \\
\hline$\delta$ total serum IgE (IU/mL, at 4 months-baseline) & $-54.83 \pm 45.73$ & $-92.0 \pm 167.2$ & 0.61 \\
\hline $\mathrm{FEV}_{1}(\%$ predicted, at 4 months) & $79.73 \pm 13.84$ & $79.42 \pm 24.66$ & 0.98 \\
\hline$\delta \mathrm{FEV}_{1}(\%$ predicted, at 4 months-baseline $)$ & $14.47 \pm 9.97$ & $3.86 \pm 12.15$ & 0.15 \\
\hline $\mathrm{FEV}_{1}$ (L, at 4 months) & $1.81 \pm 0.60$ & $1.54 \pm 0.64$ & 0.48 \\
\hline$\delta \mathrm{FEV}_{1}(\mathrm{~L}$, at 4 months-baseline) & $0.35 \pm 0.33$ & $0.05 \pm 0.21$ & 0.11 \\
\hline
\end{tabular}

Values are presented as mean $\pm \mathrm{SD}$.

ACQ: Asthma Control Questionnaire, $\mathrm{FEV}_{1}$ : forced expiratory volume in $1 \mathrm{~s}$
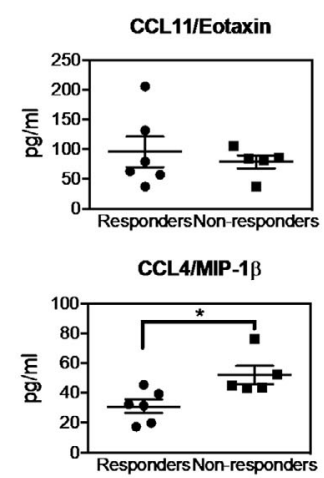

PDGF

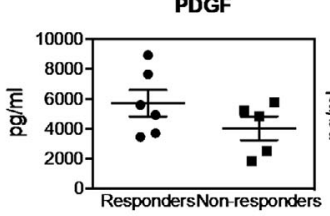

IL-4

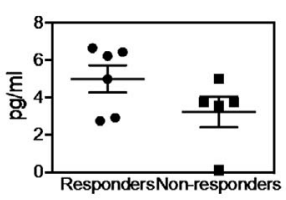

IFN-Y

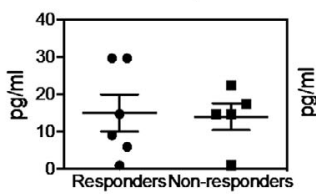

VEGF

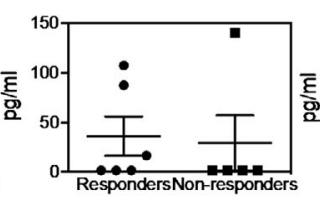

IL-5

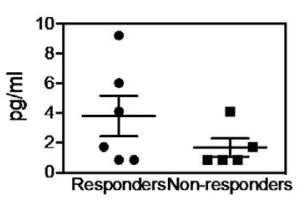

CXCL10/P-10

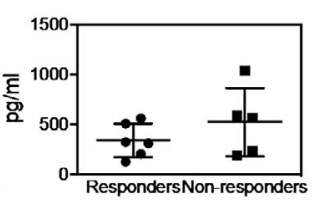

TSLP

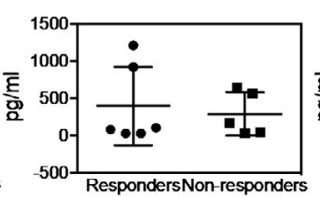

IL-9

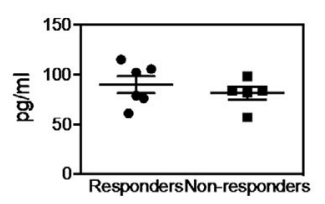

IL-12

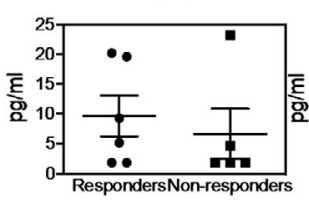

IL-33

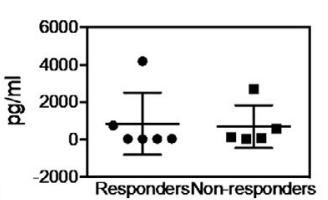

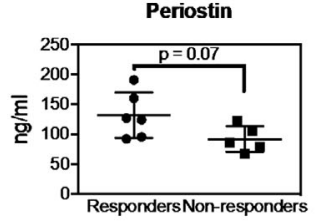

CHI3L1
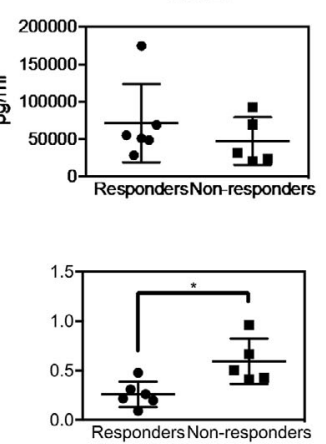

Figure 2. The baseline serum cytokine levels and the ratio of CCL4/MIP-1 $\beta$ (CCL4) to periostin in responders and non-responders to mepolizumab therapy. The baseline serum cytokine levels and the ratios are shown for responders (circles) and non-responders (squares) to mepolizumab therapy. Bars represent the mean \pm SEM. * $\mathbf{p}<0.05$.

The baseline characteristics of the patients in both subject groups are shown in Table 1 . The groups showed no significant difference in regard to age, BMI, disease duration, smoking index, baseline FeNO level, baseline blood eosinophil or neutrophil counts, baseline serum IgE level, baseline FEV1, baseline AQLQ score, baseline VAS of asthma symptoms or baseline asthma therapy, including oral corticosteroid use (Table 1). On the other hand, the clinical outcomes of the two groups seemed to closely reflect the change in the AQLQ score (Table 2). Responders showed a significantly decreased ACQ score compared to non-responders. Although the decreases in the FeNO level and blood eosinophil count, and the increases in \% predicted $\mathrm{FEV}_{1}$ and $\mathrm{FEV}_{1}$, did not reach statistical significance, the degree of decrease/ increase tended to be larger among the responders. Collectively, the change in the AQLQ score seemed to be a reasonable basis for judging the response to mepolizumab (Table 2).

\section{Responders showed a lower baseline CCL4/MIP-1 $\beta$ level in the serum}

Fig. 2 shows the baseline cytokine levels and the ratio of CCL4/MIP-1 $\beta$ to periostin. The responders showed a significantly lower level of CCL4/MIP-1 $\beta$ at baseline compared to the non-responders, while none of the other cytokines showed a statistically significant difference between the responders and non-responders (Fig. 2). It is noteworthy, however, that the responders tended to show higher levels of two Th2 cytokines, namely, periostin and IL-4. The ratio of CCL4/MIP-1 $\beta$ to periostin was significantly lower in the responders compared to the non-responders. The responders also tended to show a higher level of PDGF, although the difference was not statistically significant. 

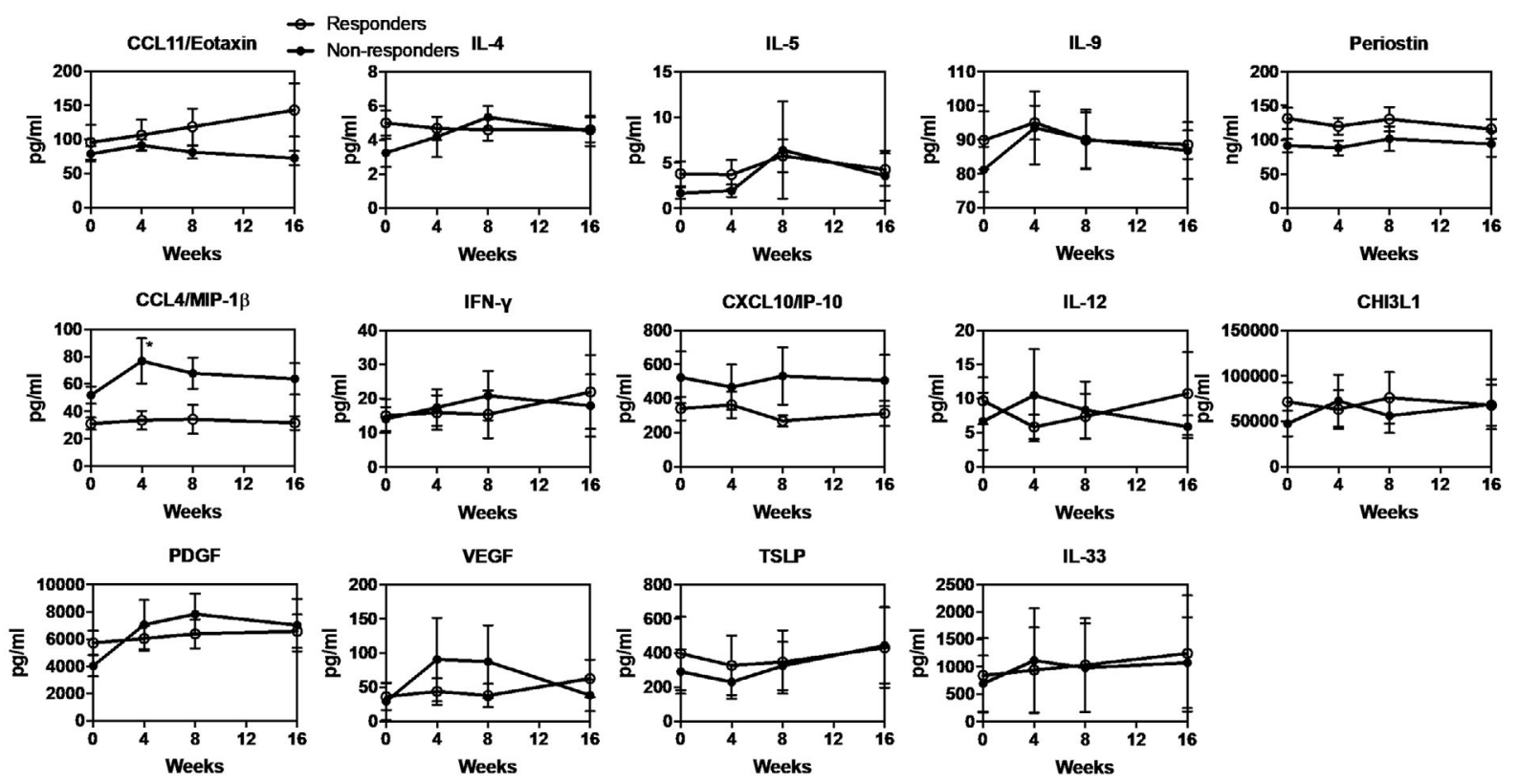

Figure 3. Change in the serum cytokine levels in both responders and non-responders who underwent mepolizumab therapy. The serum cytokine levels at baseline and after 1, 4, 8 and 16 weeks in responders (white circles) and non-responders (black circles) are shown. Bars represent the mean \pm SEM. * $\mathbf{p}<0.05$ vs. the baseline level.

\section{Changes in the serum cytokine levels during the 16 weeks of mepolizumab therapy}

Changes in the serum cytokine levels during the mepolizumab therapy were analyzed in all patients (Supplementary material). No cytokine showed a significant change from the baseline except for PDGF, which was significantly increased after 8 weeks of treatment.

When the responders and non-responders were separately analyzed for changes in the serum cytokine levels, the nonresponders showed a significantly increased level of CCL4/ MIP-1 $\beta$ after 4 weeks compared to the baseline (Fig. 3). Neither the responders nor non-responders showed a significant change in any of the other cytokines. Interestingly, the responders' CCL4/MIP-1 $\beta$ level remained low throughout the treatment (Fig. 3). In addition, the levels of CCL11/eotaxin and periostin remained high and the level of CXCL10/ IP-10 remained low throughout the treatment in the responders, although without any statistical significance (Fig. 3).

\section{Discussion}

We found that a low baseline serum level of CCL4/MIP-1 $\beta$ may predict a good response to mepolizumab as evaluated by an improvement in the AQLQ score after 4 months of treatment. In addition, the level of serum CCL4/MIP-1 $\beta$ in non-responders to mepolizumab was significantly increased at 4 weeks after the initiation of mepolizumab therapy, whereas the CCL4/MIP-1 $\beta$ level in the responders remained low throughout the treatment.

At present, there is no good biomarker for predicting responsiveness to mepolizumab other than the blood eosino- phil count, which has been shown to predict the treatment response in two large mepolizumab clinical trials $(11,13)$. The finding that the blood eosinophil count is able to predict responsiveness to mepolizumab is reasonable because mepolizumab is targeted to inhibit IL-5, one of the most powerful potentiators of eosinophils, from binding to IL-5 receptor (17). On the other hand, since the serum level of IL-5 is basically low, as demonstrated in the present study (Fig. 2), and no relationship was reported between the serum IL-5 level and the clinical parameters for asthma (18), IL-5 itself may not be a good candidate marker for predicting the response to mepolizumab therapy. It seems logical that patients with more eosinophils have eosinophilic inflammation and that blocking the activity of IL-5 by mepolizumab suppresses eosinophilic inflammation by inhibiting the activation and survival of eosinophils. However, it still seemed desirable to identify a better and more convenient marker that predicts responsiveness to mepolizumab because today we have various other powerful biological agents, i.e., omalizumab, benralizumab and dupilumab, that can be used to treat severe asthma.

It was somewhat of a surprise that a low baseline level of CCL4/MIP-1 $\beta$ would be found useful for predicting the efficacy of mepolizumab, since we measured several moreclearly understood Th1 and Th2 cytokines in the present study, i.e., IL-4, 5, 9, periostin, CCL11/eotaxin and IFN- $\gamma$. In addition, CCL4/MIP- $1 \beta$ has yet not been fully characterized in the field of asthma. CCL4/MIP-1 $\beta$ is a ligand for CC chemokine receptor type 5 (CCR5), is a potent chemokine that attracts mainly macrophages and CD4-positive $\mathrm{T}$ cells, and also works as an activator for natural killer cells (19). Its receptor, CCR5, is highly expressed on memory Th1 
cells, which are reported to play an important role in certain phenotypes of asthma as well as in mouse models of asthma (20-23). In a murine model of asthma, the expression of CCR5 was induced, and the abrogation of CCR5 was found to be effective for treating allergic inflammation, thus resulting in an improved pulmonary function. There are other ligands for CCR5, namely CCL5/RANTES and CCL3/ MIP-1 $\alpha$, which may be the main players in CCR5-activated asthma. In contrast to the field of allergy or type 2 inflammation, CCL4/MIP-1 $\beta$ has been categorized as a Th1 cytokine and it has been shown to increase during viral infections, including respiratory syncytial virus (RSV) infection (24-27). We have to note that in the present study setting, we could not find a negative correlation between the level of CCL4/MIP-1 $\beta$ and type 2 biomarkers, namely, blood eosinophils, FeNO or IgE.

Based on the knowledge that eosinophilic inflammation, for which mepolizumab is likely to be chosen, is a type 2 inflammation, we imagine that such eosinophilic inflammation would show decreased levels of Th1 cytokines, including CCL4/MIP-1 $\beta$. In fact, another Th1 cytokine, CXCL10/ IP-10, tended to be lower and Th2 cytokines such as CCL 11/eotaxin, IL-4, IL-5 and periostin tended to be higher in the responders, although without any statistical significance (Fig. 2). Thus, our present results may indicate that mepolizumab therapy is effective for type 2 inflammation-dominant asthma and that a low serum level of CCL4/MIP-1 $\beta$, a Th1 cytokine, is a reliable marker for accurate prediction of responders. In contrast, neutrophilic inflammation was reported in another phenotype of asthma (28). Although neutrophils are also potent producers of CCL4/MIP-1 $\beta$ (29), we found no difference in the number of peripheral blood neutrophils between the responders and non-responders (Table 1).

A time-course analysis of serum cytokines found that PDGF increased during mepolizumab treatment, regardless of its efficacy (Supplementary material). Due to PDGF's role in activating eosinophils $(30,31)$, anti-IL-5 therapy with mepolizumab might have had a negative feedback effect that resulted in an enhanced production of PDGF. It was interesting to find a significant elevation of serum CCL4/MIP-1 $\beta$ in the non-responders (Fig. 3). The serum level of CCL4/MIP$1 \beta$ seemed to be higher at each time point, which was therefore indicative of a non-Th2 dominant pattern of asthma in those non-responders (Fig. 3).

Some of the limitations associated with this study include the small number of participants and the fact that the study was conducted at only one hospital. Since we attempted to divide such a small population into two groups, there may have been a placebo effect or an effect arising from the baseline asthmatic condition. In addition, since we performed analyses as initially planned before conducting this research, we did not correct for multiple cytokine comparisons; accordingly, our results may have a reduced impact. A larger study needs to be performed among multiple hospitals, with more participants, to conclude that a low serum level of CCL4/MIP-1 $\beta$ is a useful and reliable biomarker for predicting responsiveness to mepolizumab. In addition, since there are several biologics for severe asthma today, the serum levels of cytokines have to be compared in patients on other drugs at baseline and after therapy in order to apply the findings to precision medicine. Larger prospective studies are necessary to further clarify the importance of CCL4/ MIP-1 $\beta$ as a predictor of a mepolizumab response and to elucidate the roles of cytokines-including CCL4/MIP-1 $\beta$-in the pathogenesis of severe asthma.

In conclusion, a low baseline serum CCL4/MIP-1 $\beta$ level predicted a good response to mepolizumab therapy in eosinophilic severe asthma, and the serum level of PDGF increased in all patients after 8 weeks of therapy. Today, several biological drugs are available for the treatment of eosinophilic severe asthma, and there is a need for reliable biomarkers to achieve precision medicine. Larger studies are required to elucidate the clinical usefulness and establish the cut-off values for serum CCL4/MIP-1 $\beta$ to be used in clinical settings.

\section{The authors state that they have no Conflict of Interest (COI).}

\section{Acknowledgement}

The authors thank Ms. Sayaka Igarashi for her skilled technical assistance. Thanks are also extended to Ms. Mariko Yoshizawa and Ms. Taeko Kawabe for their excellent secretarial work.

\section{References}

1. Chung KF, Wenzel SE, Brozek JL, et al. International ERS/ATS guidelines on definition, evaluation and treatment of severe asthma. Eur Respir J 43: 343-373, 2014.

2. Hekking PP, Wener RR, Amelink M, Zwinderman AH, Bouvy ML, Bel EH. The prevalence of severe refractory asthma. J Allergy Clin Immunol 135: 896-902, 2015.

3. Chen S, Golam S, Myers J, Bly C, Smolen H, Xu X. Systematic literature review of the clinical, humanistic, and economic burden associated with asthma uncontrolled by GINA Steps 4 or 5 treatment. Curr Med Res Opin 34: 2075-2088, 2018.

4. Corren J. New Targeted Therapies for Uncontrolled Asthma. J Allergy Clin Immunol Pract 7: 1394-1403, 2019.

5. Manka LA, Wechsler ME. Selecting the right biologic for your patients with severe asthma. Ann Allergy Asthma Immunol 121: 406413, 2018.

6. Ohta K, Nagase H, Suzukawa M, Ohta S. Antibody therapy for the management of severe asthma with eosinophilic inflammation. Int Immunol 29: 337-343, 2017.

7. Hanania NA, Wenzel S, Rosen K, et al. Exploring the effects of omalizumab in allergic asthma: an analysis of biomarkers in the EXTRA study. Am J Respir Crit Care Med 187: 804-811, 2013.

8. Suzukawa M, Matsumoto $H$, Ohshima $N$, et al. Baseline serum CXCL10 and IL-12 levels may predict severe asthmatics' responsiveness to omalizumab. Respir Med 134: 95-102, 2018.

9. Boulet LP, Reddel HK, Bateman E, Pedersen S, FitzGerald JM, O'Byrne PM. The Global Initiative for Asthma (GINA): 25 years later. Eur Respir J 54: 2019.

10. Holguin F, Cardet JC, Chung KF, et al. Management of severe asthma: a European Respiratory Society/American Thoracic Society guideline. Eur Respir J 55: 1900588, 2020.

11. Pavord ID, Korn S, Howarth $P$, et al. Mepolizumab for severe eosinophilic asthma (DREAM): a multicentre, double-blind, 
placebo-controlled trial. Lancet 380: 651-659, 2012.

12. Bel EH, Wenzel SE, Thompson PJ, et al. Oral glucocorticoidsparing effect of mepolizumab in eosinophilic asthma. N Engl J Med 371: 1189-1197, 2014.

13. Ortega HG, Liu MC, Pavord ID, et al. Mepolizumab treatment in patients with severe eosinophilic asthma. $\mathrm{N}$ Engl $\mathrm{J}$ Med 371: 1198-1207, 2014.

14. Ortega HG, Yancey SW, Mayer B, et al. Severe eosinophilic asthma treated with mepolizumab stratified by baseline eosinophil thresholds: a secondary analysis of the DREAM and MENSA studies. Lancet Respir Med 4: 549-556, 2016.

15. Yancey SW, Keene ON, Albers FC, Ortega H, Bates S, Bleecker ER, et al. Biomarkers for severe eosinophilic asthma. J Allergy Clin Immunol 140: 1509-1518, 2017.

16. Arakawa S, Suzukawa M, Ohshima N, et al. Expression of Siglec8 is regulated by interleukin-5, and serum levels of soluble Siglec8 may predict responsiveness of severe eosinophilic asthma to mepolizumab. Allergol Int 67S: S41-S44, 2018.

17. Smith DA, Minthorn EA, Beerahee M. Pharmacokinetics and pharmacodynamics of mepolizumab, an anti-interleukin-5 monoclonal antibody. Clin Pharmacokinet 50: 215-227, 2011.

18. ten Hacken NH, Oosterhoff Y, Kauffman HF, et al. Elevated serum interferon-gamma in atopic asthma correlates with increased airways responsiveness and circadian peak expiratory flow variation. Eur Respir J 11: 312-316, 1998.

19. Menten P, Wuyts A, Van Damme J. Macrophage inflammatory protein-1. Cytokine Growth Factor Rev 13: 455-481, 2002.

20. Bisset LR, Schmid-Grendelmeier P. Chemokines and their receptors in the pathogenesis of allergic asthma: progress and perspective. Curr Opin Pulm Med 11: 35-42, 2005.

21. Berce V, Repnik K, Potocnik U. Association of CCR5-delta32 mutation with reduced risk of nonatopic asthma in Slovenian children. J Asthma 45: 780-784, 2008.

22. Suzaki Y, Hamada K, Nomi T, et al. A small-molecule compound targeting CCR5 and CXCR3 prevents airway hyperresponsiveness and inflammation. Eur Respir J 31: 783-789, 2008.

23. Walker JK, Ahumada A, Frank B, et al. Multistrain genetic com- parisons reveal CCR5 as a receptor involved in airway hyperresponsiveness. Am J Respir Cell Mol Biol 34: 711-718, 2006.

24. Schrum S, Probst P, Fleischer B, Zipfel PF. Synthesis of the CCchemokines MIP-1alpha, MIP-1beta, and RANTES is associated with a type 1 immune response. J Immunol 157: 3598-3604, 1996.

25. Zhang Y, Luxon BA, Casola A, Garofalo RP, Jamaluddin M, Brasier AR. Expression of respiratory syncytial virus-induced chemokine gene networks in lower airway epithelial cells revealed by cDNA microarrays. J Virol 75: 9044-9058, 2001.

26. Haeberle HA, Nesti F, Dieterich HJ, Gatalica Z, Garofalo RP. Perflubron reduces lung inflammation in respiratory syncytial virus infection by inhibiting chemokine expression and nuclear factorkappa B activation. Am J Respir Crit Care Med 165: 1433-1438, 2002.

27. Salentin R, Gemsa D, Sprenger H, Kaufmann A. Chemokine receptor expression and chemotactic responsiveness of human monocytes after influenza A virus infection. J Leukoc Biol 74: 252-259, 2003.

28. Moore WC, Hastie AT, Li X, et al. Sputum neutrophil counts are associated with more severe asthma phenotypes using cluster analysis. J Allergy Clin Immunol 133: 1557-1563. e5, 2014.

29. Chiba K, Zhao W, Chen J, et al. Neutrophils secrete MIP-1 beta after adhesion to laminin contained in basement membrane of blood vessels. Br J Haematol 127: 592-597, 2004.

30. Bach MK, Brashler JR, Stout BK, Johnson HG, Sanders ME. Platelet-derived growth factor can activate purified primate, phorbol myristate acetate-primed eosinophils. Int Arch Allergy Appl Immunol 94: 167-168, 1991.

31. Bach MK, Brashler JR, Stout BK, et al. Activation of human eosinophils by platelet-derived growth factor. Int Arch Allergy Immunol 97: 121-129, 1992.

The Internal Medicine is an Open Access journal distributed under the Creative Commons Attribution-NonCommercial-NoDerivatives 4.0 International License. To view the details of this license, please visit (https://creativecommons.org/licenses/ by-nc-nd/4.0/).

(C) 2020 The Japanese Society of Internal Medicine Intern Med 59: 2849-2855, 2020 